\title{
Formas farmacêuticas de liberação modificada: polímeros hidrifílicos
}

\author{
Carla Martins Lopes*, José Manuel Sousa Lobo, Paulo Costa
}

Serviço de Tecnologia Farmacêutica, Faculdade de Farmácia, Universidade do Porto, Portugal

\footnotetext{
*Correspondência:

C. M. Lopes

Serviço de Tecnologia Farmacêutica da Faculdade de Farmácia da Universidade do Porto

Rua Aníbal Cunha, 164 4050-047 - Porto-Portugal E-mail: clopes@ff.up.pt
}

Os sistemas de liberação de fármacos são parte integrante da investigação farmacêutica. A maioria dos sistemas de liberação oral de fármacos é baseada em matrizes poliméricas. Nas duas décadas passadas, as matrizes hidrofilicas tornaram-se muito populares na formulação de formas farmacêuticas de liberação modificada. A escolha do polímero hidrofilico na formulação da matriz pode fornecer uma combinação apropriada dos mecanismos de intumescimento, de dissolução ou de erosão e determinam a cinética de liberação in vitro. As matrizes de intumescimento são sistemas monolíticos preparados pela compressão de mistura de um polímero hidrofilico e de um fármaco. Elas representam sistemas da liberação em que os vários mecanismos podem ser adaptados ao programa de liberação. O sucesso desses sistemas está relacionado com a tecnologia de fabricação e com as características fisicas e físico-químicas do polímero, responsáveis pelo mecanismo de liberação.
Unitermos

- Liberação modificada

- Polímeros matriciais

- Matrizes hidrofílicas

\section{INTRODUÇÃO}

A tecnologia associada à modificação da liberação de fármacos, ou outras substâncias bioativas, a partir de preparações farmacêuticas sofreu um incremento notório nas últimas décadas na tentativa de maximizar as vantagens inerentes às formas farmacêuticas de liberação controlada (Das, Das, 2003). Ampla variedade de sistemas, visando condicionar a velocidade e o local de liberação dos fármacos, tem sido objeto de investigação na área da indústria farmacêutica. Entre estes sistemas estão incluídos os lipossomas, as bombas osmóticas, os revestimentos entéricos, os sistemas transdérmicos, os pró-fármacos, os sistemas matriciais poliméricos, entre outros.

De maneira particular, a utilização de sistemas matriciais constituídos por diversos tipos de polímeros é opção interessante, sendo uma das estratégias mais empregadas quando do desenvolvimento de uma formulação oral de liberação modificada devido às vantagens inerentes a estes sistemas: versatilidade, eficácia, baixo custo e produção que recorre a equipamentos e técnicas convencionais. Além disso a utilização de sistemas matriciais permite a incorporação de quantidades relativamente elevadas de fármacos. Do ponto de vista tecnológico, um sistema matricial pode ser definido como sistema que controla a liberação da(s) substância(s) ativa(s), molecularmente dispersa(s) ou dissolvida(s) num suporte resistente à desintegração (polímero ou agente formador da matriz).

A classificação dos sistemas matriciais (Buri, 1987) leva em consideração diversos critérios, especificamente a estrutura da matriz, a cinética de liberação (idealmente de ordem zero), os mecanismos para controlar a liberação 
(erosão, difusão, intumescimento), a natureza química e as propriedades dos materiais utilizados. A erosão, a difusão e o intumescimento das matrizes são os vários mecanismos pelos quais os sistemas matriciais podem controlar a liberação das substâncias ativas. A predominância de um destes mecanismos depende invariavelmente das propriedades do polímero empregado no sistema. De um modo geral, quando as estruturas matriciais entram em contacto com o meio de dissolução (ou fluído biológico) podem manter a sua estrutura mais ou menos constante ao longo de todo o processo de dissolução ou podem sofrer um fenômeno de intumescimento ("swelling") e, posteriormente, de erosão.

\section{AGENTES POLIMÉRICOS}

A melhoria no desenvolvimento de sistemas de liberação modificada depende estritamente da seleção de um agente apropriado capaz de controlar a liberação do fármaco, sustentar a ação terapêutica ao longo do tempo e/ ou de liberar o fámaco ao nível de um determinado tecido ou órgão alvo. Dentro das várias opções, os polímeros são agentes versáteis e promissores para exercer tal função.

Nos sistemas farmacêuticos matriciais o fármaco está incluído num sistema formado por cadeias de uma ou várias substâncias químicas polimerizadas, que funcionam como agentes moduladores da liberação. Segundo Lordi, os materiais retardantes que podem ser utilizados para formular comprimidos matriciais (Lordi, 1970) dividem-se nos seguintes grupos:

- Matrizes insolúveis e inertes (ex.: etilcelulose, polietileno, cloreto de polivinila, etc).

- Matrizes insolúveis em água e erodíveis (ex.: triglice- rídeos, cera de carnaúba, óleo de rícino hidrogenado, etc);

- Polímeros hidrofílicos (ex.: metilcelulose, hidroxipropilmetilcelulose, hidroxietilcelulose, alginato de sódio, carboximetilcelulose sódica, carbômeros, etc);

Neste contexto, a palavra inerte refere-se não à inércia farmacológica, mas sim à inércia relativamente ao comportamento mecanístico do polímero quando na presença da água, mantendo praticamente constante a sua estrutura.

As matrizes inertes são constituídas por polímeros insolúveis, que originam estruturas porosas nas quais o fármaco está disperso, mantendo a mesma superfície aparente (interface sólido/líquido de dissolução) ao longo de toda a etapa de dissolução. Os comprimidos preparados com polímeros inertes formam sistemas que não se alteram ao longo do trato gastrintestinal, sendo eliminados praticamente intactos. A dissolução do fármaco nas matrizes inertes verifica-se após penetração do líquido de dissolução nos poros do sistema matricial, seguindo-se etapa de difusão lenta do fármaco dissolvido nos canalículos (Salomon, Doelker,1980), como pode ser visto na Figura 1. O passo limitante da velocidade de liberação dos fármacos a partir deste tipo de sistemas é a penetração do líquido de dissolução na matriz, sendo a permeação da água promovida pela adição de agentes molhantes.

Nas matrizes hidrofóbicas ou lipídicas o controle da liberação da substância ativa ocorre essencialmente por mecanismos de difusão através dos poros ou por erosão, prevalecendo um ou outro mecanismo de acordo com as propriedades do fármaco e do excipiente utilizado.

As matrizes lipídicas são, muitas vezes, incluídas na classe das matrizes inertes, visto que ambas, quando em

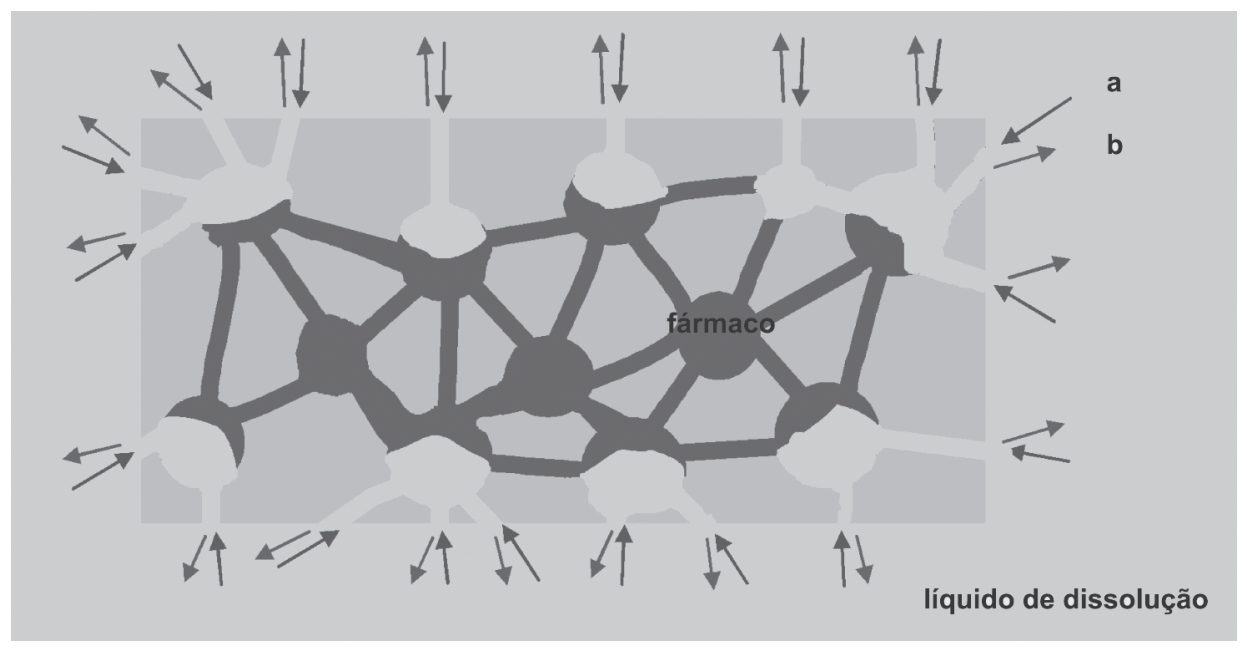

FIGURA 1 - Etapas de liberação dos fármacos em sistemas matriciais inertes ou hidrofóbicos: a) penetração do líquido de dissolução nos poros do sistema matricial; b) difusão lenta pelos canalículos do fármaco dissolvido até o exterior. 
contacto com a água, não alteram a sua estrutura e apresentam mecanismos de liberação dos fármacos semelhantes.

Os numerosos agentes gelificantes usados nas investigações das matrizes hidrofílicas podem dividir-se em três classes (Buri, Doelker, 1980):

- Derivados da celulose (ex.: metilcelulose, hidroxipropilmetilcelulose, hidroxietilcelulose, hidroxipropilcelulose, carboximetilcelulose sódica);

- Polímeros não-celulósicos naturais ou semi-sintéticos (ex.: agar-agar, alginatos, gomas, quitosana)

- Polímeros do ácido acrílico (exemplo: carbômeros).

\section{MATRIZES HIDROFÍLICAS - DERIVADOS CELULÓSICOS}

Apesar de muitos sistemas poliméricos poderem ser utilizados na preparação de formas farmacêuticas orais de liberação modificada, os polímeros hidrodispersíveis, como os éteres da celulose, são talvez o grupo mais freqüentemente empregado neste tipo de formulações.

Alguns autores consideram que os polímeros que apresentam a capacidade de intumescimento e que são insolúveis em água devem ser designados hidrogéis (Peppas et al., 2000), enquanto que os hidrossolúveis devem ser designados polímeros hidrofílicos ou hidrodispersíveis.

Os derivados da celulose apresentam ampla aplicação na indústria farmacêutica, como aglutinantes, no revestimento de comprimidos e, mais recentemente, como agentes moduladores da liberação na preparação de comprimidos de liberação prolongada (Pham, Lee, 1994; Gao et al., 1996; Eyjolfsson, 1999).

Um dos derivados da celulose mais usados (desde o início dos anos de 1960) como retardante da liberação de fármacos, em formulações orais, é a hidroxipropilmetilcelulose (HPMC). Esta ampla utilização de matrizes de HPMC deve-se à existência de grande variedade deste polímero, que se classifica de acordo com a sua viscosidade e a proporção entre os grupos substituintes (grupos metoxila e hidroxipropila). O grau de hidratação deste polímero hidrossolúvel depende das características físicoquímicas, como a massa molecular, o grau de substituição e a viscosidade. A fórmula estrutural está representada na Figura 2.

Uma das características mais importantes deste polímero hidrofílico, no que se refere à sua aplicação em sistemas de liberação modificada, é a capacidade de intumescimento/relaxamento, a qual exerce efeito pronunciado na cinética de liberação do fármaco incorporado. A sua popularidade deve-se, também, a outras características, como a natureza não-tóxica e não-iônica do polímero (não apresentando problemas de compatibilidade), a capa-

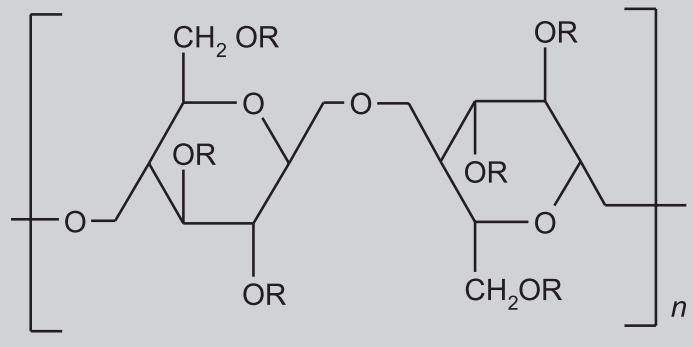

FIGURA 2 - Estrutura química da hidroxipropilmetilcelulose (HPMC).

cidade de incorporar elevadas quantidades de substâncias ativas, a simplicidade, a rapidez e a economia da tecnologia associada à sua preparação e à possibilidade de fabricação de comprimidos matriciais por compressão direta, apresentando boas características de compressão sem necessidade de etapa prévia de granulação (Feely, Davis, 1988a; Wan et al., 1991; Skoug et al., 1993; Pham, Lee, 1994; Tahara et al., 1995, Rodriguez et al., 2000). A hidratação da HPMC não é afetada pela variação natural do $\mathrm{pH}$ ao longo do trato gastrintestinal. A HPMC parece exercer certo poder tampão, podendo tornar os sistemas independentes do $\mathrm{pH}$ (Feely, Davis, 1988b). Dos vários tipos de HPMC, os mais utilizados, para preparar sistemas matriciais de intumescimento são os que apresentam um grau de viscosidade elevado - HPMC 2208, HPMC 2906 e HPMC 2910, de acordo com as especificações da USP XXV/NF XX.

Existe na literatura várias publicações que citam a influência da proporção da matriz hidrofílica nas características físicas e de liberação de comprimidos (Ford et al., 1985, 1987; Sheskey et al. 1992, 1994). O mecanismo pelo qual a HPMC atua e o seu desempenho no sistema matricial resultante têm sido objeto de diversas investigações. Alguns dos parâmetros investigados incluem a taxa de penetração da água no sistema (Wan et al., 1991), a velocidade de hidratação e a extensão do intumescimento do polímero (Wan et al., 1995; Colombo et al., 1999).

Foram propostos outros polímeros, apresentando um comportamento comparável à HPMC, como agentes de intumescimento em matrizes hidrofílicas. O óxido de polietileno (PEO) foi introduzido como agente alternativo para matrizes de intumescimento. Outros polímeros, como o carbopol ou as gomas naturais, estão também disponíveis, mas são sensíveis às variações de $\mathrm{pH}$.

Em geral, a liberação dos fármacos incorporados em sistemas constituídos por polímeros hidrofílicos baseia-se na transição do estado vítreo ("glassy") para o estado maleável ("rubbery”), resultante da penetração da água na 
matriz (Colombo et al., 1996; Juang, Storey, 2003). Um polímero, consoante a temperatura a que está submetido, apresenta-se no estado vítreo ou no estado maleável. A temperatura de transição vítrea ( $\mathrm{Tg}$ ) dos polímeros hidrofílicos é influenciada por vários fatores, dos quais se destaca a presença de líquidos plastificantes. As moléculas desses líquidos são, normalmente, de dimensões reduzidas e ficam retidas entre as cadeias poliméricas, afastando-as uma das outras, o que reduz as forças de atração intermoleculares polímero - polímero, aumentando a mobilidade das cadeias. Assim, quando um sistema matricial contém um polímero de intumescimento e entra em contacto com a água (a umidade é considerada um plastificante) ocorre mudança do estado vítreo (estado configuracional altamente emaranhado) para um estado maleável, o que está associado com o processo de intumescimento/relaxamento (Figura 3). A temperatura de transição vítrea varia de polímero para polímero (Costa, Sousa Lobo, 1999) e depende da temperatura e das interações termodinâmicas do sistema polímero-água (Colombo et al., 2000).

\section{Mecanismos de liberação dos fármacos a partir de matrizes hidrofílicas}

A HPMC é um polímero hidrofílico linear e os trabalhos realizados demonstram que os seus efeitos se manifestam principalmente pela sua capacidade de, após hidratação, intumescer e formar uma camada gelatinosa à superfície do comprimido. Esta funciona como barreira à rápida liberação do fármaco, controlando quer a penetração de água, quer a velocidade de liberação da substância ativa (Colombo et al., 2000). A velocidade de penetração da água no sistema matricial condiciona o modo de liberação do fármaco. Em concentrações muito elevadas, as cadeias lineares de HPMC formam um emaranhado, originando uma camada gelatinosa bastante consistente. Em concentrações muito baixas, estes géis celulósicos apresentam viscosidade muito baixa, permitindo a liberação quase imediata dos fármacos.

A velocidade de liberação do fármaco, no caso de comprimidos matriciais constituídos à base de polímeros hidrofílicos, é condicionada por um ou mais dos seguintes mecanismos cinéticos (Ranga Rao, Davi, 1988):

- Transporte do meio de dissolução para a matriz polimérica;

- Intumescimento ("swelling") do polímero com formação de uma camada de gel;

- Difusão do fármaco através da camada de polímero intumescido;

- Erosão do polímero intumescido.

A etapa de liberação dos fármacos a partir de sistemas farmacêuticos de matriz hidrofílica envolve as várias fases representadas na Figura 4.

Numa primeira fase, as matrizes hidrofílicas (1), quando em contato com o meio de dissolução ou com o fluido aquoso gastrintestinal, absorvem água, sobretudo através dos poros do sistema matricial. Após a hidratação do sistema, com conseqüente liberação imediata do fármaco existente à superfície do comprimido, ocorre o

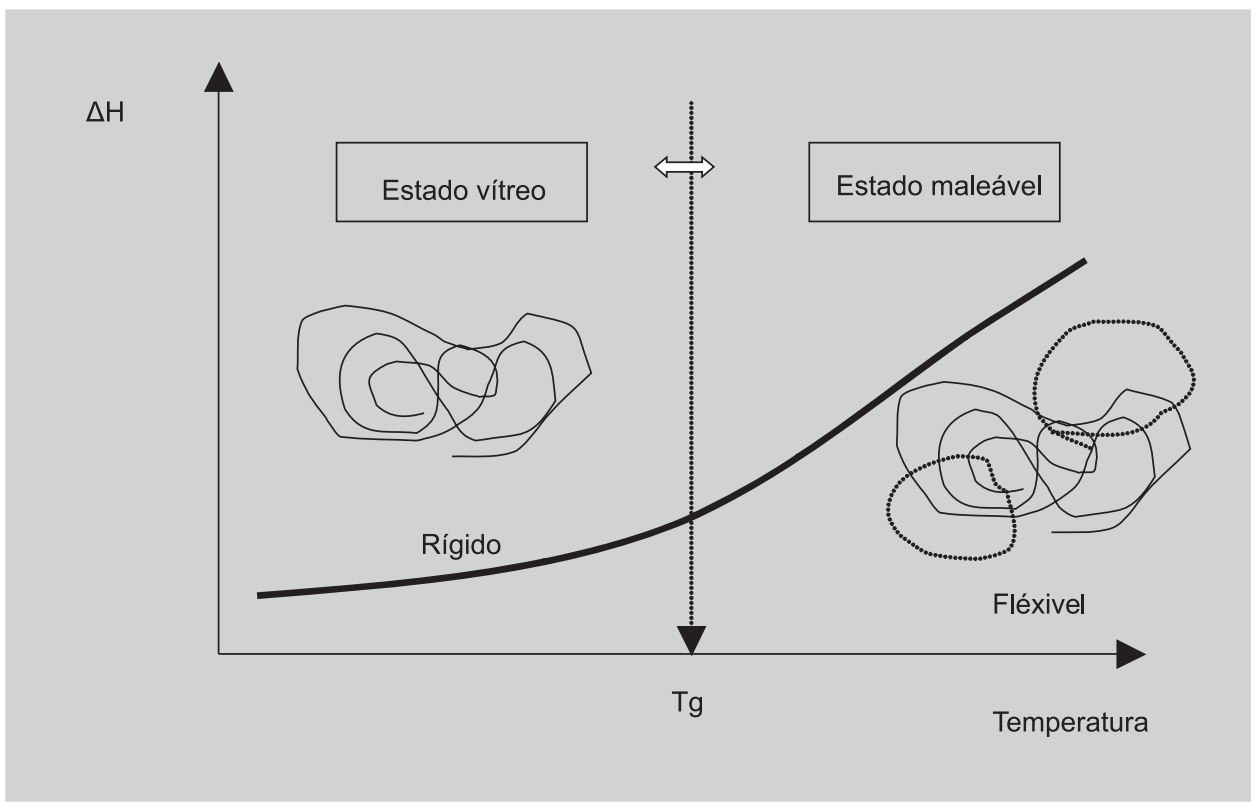

FIGURA 3 - Temperatura de transição vítrea (Tg) do estado vítreo para o estado maleável. 


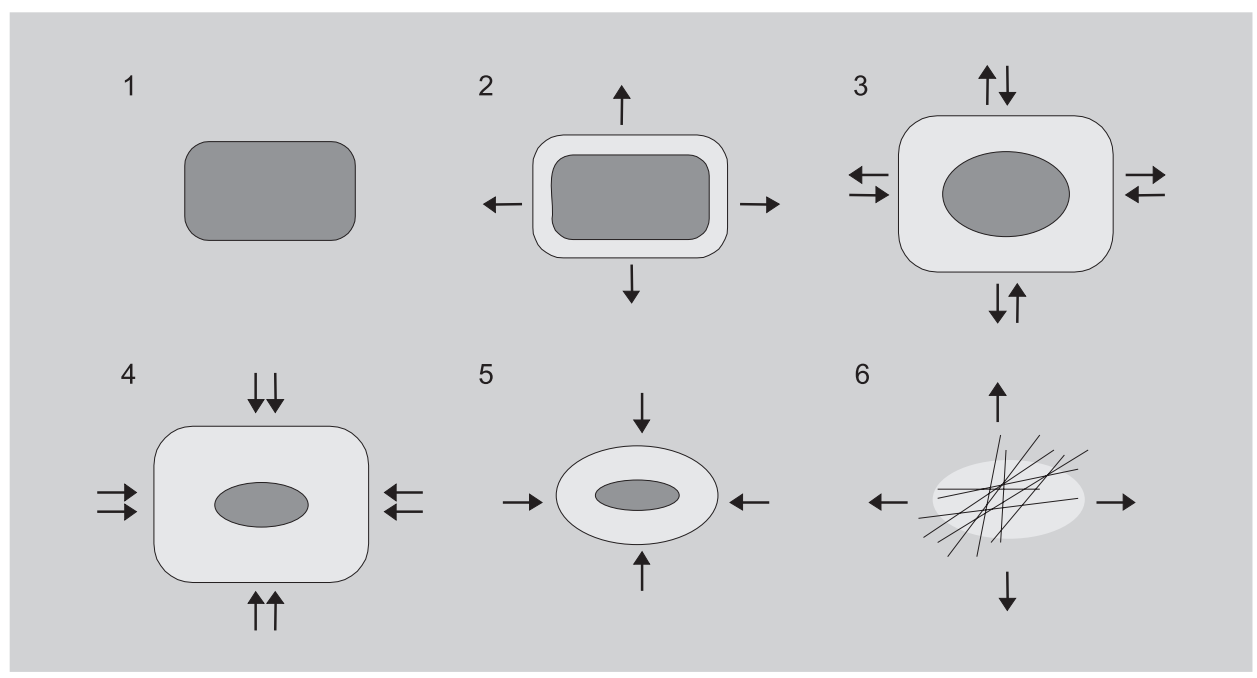

FIGURA 4 - Alterações observadas nos sistemas matriciais hidrofílicos que intumescem e sofrem erosão: 1) matriz no estado seco; 2) início da hidratação e intumescimento da matriz; 3 e 4) aumento da hidratação e intumescimento da matriz, diminuição do núcleo seco e início da erosão das cadeias poliméricas; 5) aumento da erosão das cadeias poliméricas; 6) separação das cadeias poliméricas com libertação rápida do fármaco restante.

intumescimento/relaxamento das cadeias poliméricas, e forma-se uma camada gelatinosa de polímero (estado maleável) à volta do núcleo seco do comprimido (2).

A água continua a penetrar na matriz, mas agora sobretudo através da camada gelificada, que, lentamente, se vai formando. À medida que o núcleo seco fica hidratado, a camada exterior gelificada sofre erosão. Estes dois fenômenos ocorrem simultaneamente e o sistema matricial mantém um volume mais ou menos constante (3 e 4).

Quando a penetração da água na matriz gelificada excede um valor crítico de concentração (concentração na qual as interações entre a água e o polímero aumentam com conseqüente redução das interações polímero - polímero), as cadeias poliméricas começam a se separar, alargando os espaços onde a difusão do fármaco ocorre. Nesta fase, a taxa de hidratação diminui relativamente à taxa de erosão(5). As cadeias poliméricas dispersam-se na camada mais externa, resultando em aumento da taxa de erosão.

Em conseqüência do aumento da distância entre as cadeias poliméricas, estas deixam de estar interligadas entre si, separando-se com subseqüente desintegração total do sistema (6).

\section{Frentes de movimentação nas matrizes hidrofílicas}

O comportamento de intumescimento das matrizes hidrofílicas, resultante de um processo de relaxamento das cadeias poliméricas quando em contato com o meio aquoso, é mecanicamente descrito pela formação de "frentes". Neste contexto, a palavra "frente" indica o local da matriz no qual se verifica nítida mudança das condições físicas
(Bettini et al., 1994, 1997, 1998; Colombo et al., 1995, 1996). Numa observação macroscópica do processo de intumescimento progredindo do centro para a matriz, identifica-se três frentes: a frente de intumescimento, a de difusão e a de erosão. Estas três frentes estão representadas esquematicamente na Figura 5.

A frente de intumescimento ("swelling front") corresponde à interface polímero no estado seco/camada gelificada (estado maleável), é na realidade a frente de penetração da água. A HPMC presente na zona 1 encontra-se no estado seco, possuindo temperatura de transição vítrea que se localiza entre os 154 e os $184{ }^{\circ} \mathrm{C}$ (Doelker, 1993), acima da temperatura experimental $\left(37^{\circ} \mathrm{C}\right)$. Nesta frente verifica-se a ocorrência dos fenômenos de hidratação da matriz, de intumescimento e a coalescência das partículas individuais do polímero. $\mathrm{O}$ intumescimento das matrizes de HPMC pode ser atribuído à ruptura das ligações de hidrogênio entre as cadeias do polímero (Fyfe, Blazek, 1997). Quando a água penetra no HPMC sólido, funciona como agente plastificante, inserindo-se nas ligações de hidrogênio existentes entre as cadeias poliméricas adjacentes. À medida que aumentam as interações água - polímero, as forças de interação entre as cadeias poliméricas diminuem. A água que penetra no sistema preenche os espaços entre as cadeias poliméricas e difunde-se para as regiões mais densas do polímero, "forçando" as restantes cadeias do polímero a se separar. Conseqüentemente, as cadeias ganham liberdade rotacional e começam a ocupar mais espaço, ficando num novo estado solvatado. Este novo sistema (HPMC no estado hidratado) possui temperatura de transição vítrea inferior à temperatura experimental, apre- 


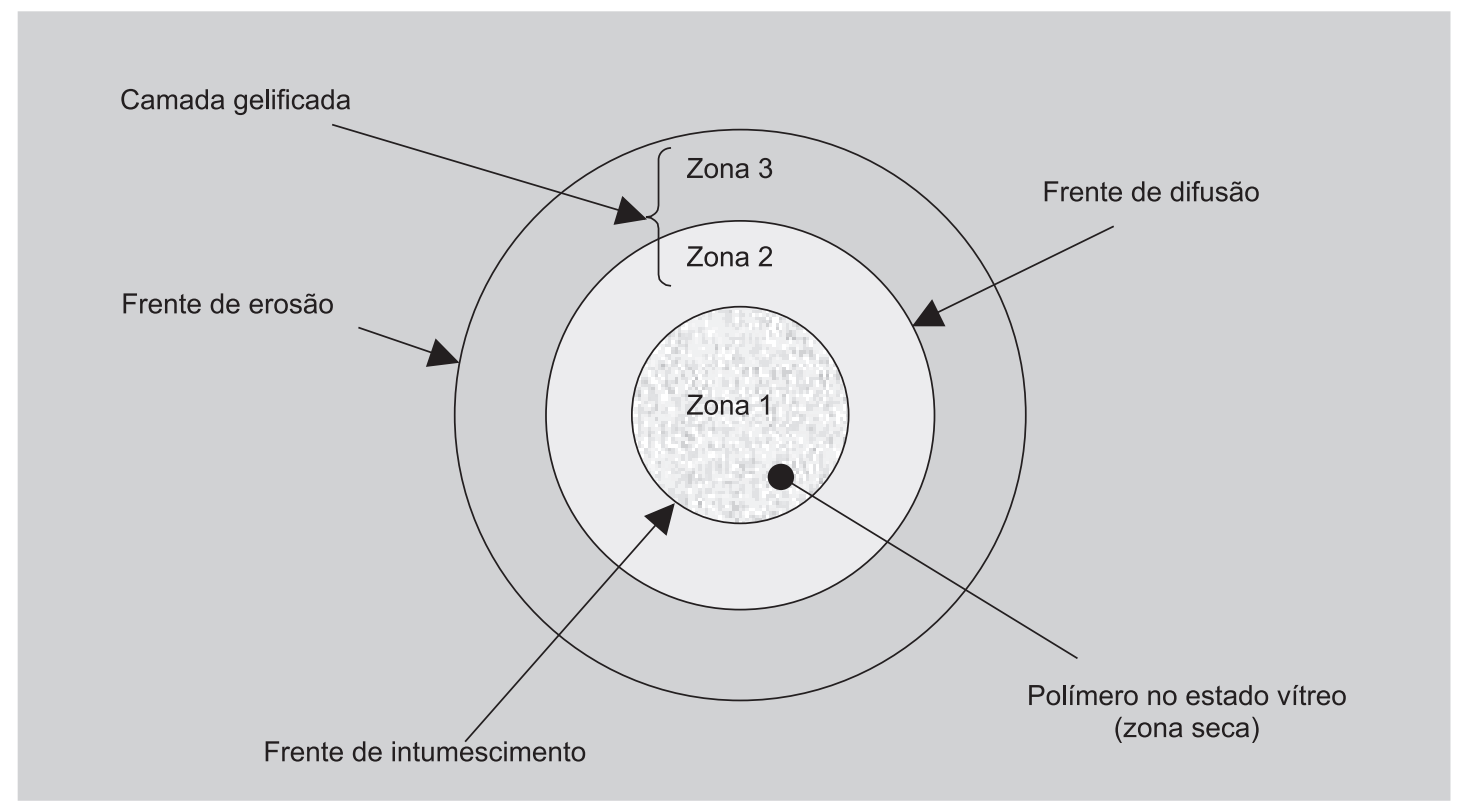

FIGURA 5 - Esquema ilustrativo das frentes de movimento (intumescimento, difusão e erosão) durante a liberação de fármacos a partir de sistemas matriciais intumescíveis: zona 1 - polímero no estado seco, zona 2 e 3: polímero no estado de maleável (ou de gel).

sentando-se no estado maleável. Na zona 1, a mobilidade macromolecular é muito baixa, conduzindo a baixas taxas de difusão da água (da ordem dos $10^{-16} \mathrm{~m}^{2} / \mathrm{s}$ à temperatura de $37^{\circ} \mathrm{C}$ ) (Wesselingh, 1993).

A segunda frente, chamada frente de difusão, situase entre a frente de intumescimento e a frente de erosão na camada gelificada. Da camada de gel fazem parte duas zonas distintas, zonas 2 e 3 da Figura 5. A principal diferença entre estas zonas é o estado de dissolução do fármaco. Na zona 2, o fármaco está presente no estado não dissolvido podendo, alguma parte dele, existir simultaneamente na forma dissolvida. Na zona 3, o fármaco encontra-se dissolvido. Assim, a frente de difusão relaciona-se com a velocidade de dissolução do fármaco. O fármaco dissolvido difunde-se em direção à frente de erosão.

Uma terceira frente, ou frente de erosão, estabelece simplesmente a ligação entre a superfície da matriz e o meio de dissolução e corresponde à interface camada de gel/meio de dissolução (água ou solução tampão). Nesta zona da estrutura matricial verifica-se o afastamento e a solubilização das cadeias poliméricas e a subseqüente liberação do fármaco.

Nas zonas 2 e 3 a mobilidade das cadeias poliméricas sofre um incremento acentuado, quando comparada com a da zona 1, conduzindo a velocidades de difusão da água muito mais elevadas, da mesma ordem de grandeza da água pura $\left(10^{-10} \mathrm{~m}^{2} / \mathrm{s}\right.$ a $\left.23{ }^{\circ} \mathrm{C}\right)$ (Gao, Fagerness, 1995).

A posição destas frentes na matriz polimérica pode ser medida, permitindo calcular três importantes parâmetros do processo de intumescimento/dissolução: a velocidade de penetração da água, a velocidade de dissolução do fármaco e a velocidade de erosão da matriz polimérica (Colombo et al., 1995).

\section{Espessura e estrutura da camada de gel}

Tal como foi referido anteriormente, os sistemas de matriciais de intumescimento são ativados pela absorção de água, sendo a liberação do fármaco controlada pela interação entre a água, o polímero e o fármaco. O elemento central do mecanismo de liberação é a formação da camada de gel à volta da matriz (Colombo et al., 2000).

A movimentação das frentes, anteriormente descritas, condiciona toda a dinâmica relacionada com a formação, a estrutura e a espessura da camada de gel. Durante a liberação do fármaco, a camada gelatinosa é exposta a mudanças contínuas da sua estrutura e espessura.

Relativamente ao crescimento da camada gelificada do polímero, como se pode verificar na Figura 6, ocorrem três fases em todo este processo.

A espessura da camada de gel é definida pela frente de erosão e pela frente de intumescimento. A espessura da camada de gel é função da quantidade de água que penetra no sistema, da taxa de desintegração das cadeias poliméricas e da transferência, em termos de massa, do fármaco e do polímero do sistema para o meio de dissolução.

Numa fase inicial (a), verifica-se um aumento rápido da espessura da camada de gel, pois a taxa de absorção de água é superior à desintegração das cadeias de 


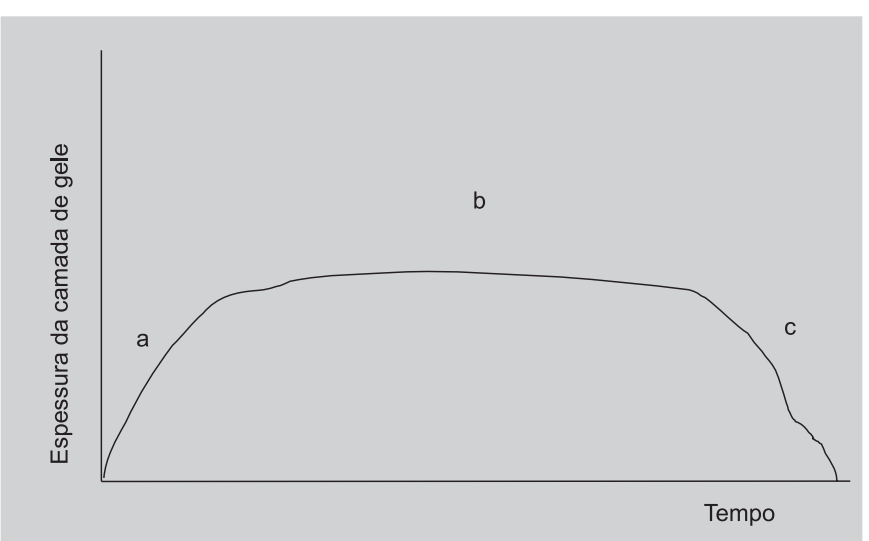

FIGURA 6 - Espessura da camada de gel (estado maleável) em função do tempo: a) aumenta devido ao intumescimento, b) mantém-se constante motivado pela sincronização da taxa de intumescimento e da taxa de erosão e c) diminui em virtude do aumento da taxa de erosão e diminuição da taxa de intumescimento.

polímero, com seu conseqüente intumescimento. A penetração do solvente é o mecanismo mais rápido.

Numa segunda fase (b), à medida que o núcleo seco se hidrata, verificam-se pequenas mudanças na densidade da camada de gel, existindo uma sincronização entre os fenómenos de intumescimento e a taxa de desintegração das cadeias poliméricas.

Numa fase final (c), quando todo o polímero está hidratado na forma gelatinosa, ocorre apenas a desintegração das suas cadeias poliméricas; neste caso, o fenômeno de dispersão prevalece em relação aos outros fenômenos e a espessura diminui até desaparecer completamente a matriz gelificada.

Com relação à mudança na estrutura da camada de gel, as cadeias poliméricas passam pelos três tipos diferentes de rearranjos espaciais representados na Figura 7. A difusão do fármaco é obviamente afetada pelo tipo de configuração molecular (Narasimhan, Peppas, 1997).

Antes de iniciar a dissolução (quando o polímero está em quantidade superior ao fármaco), predominam as interações polímero - polímero e ocorre o primeiro tipo de configuração, em que as cadeias poliméricas estão compactadas e completamente integradas umas nas outras, - trata-se do tipo concentrado ou compactado, designado por "swollen rubbery layer" (Figura 7 A). Nesta fase o fármaco encontra-se também aprisionado entre estas cadeias poliméricas, sendo mais lenta a sua liberação.

O início da etapa de dissolução na camada limite de difusão conduz ao segundo tipo de rearranjo espacial, designado por semidiluído (Figura 7 B). Este tipo corresponde à camada limite de difusão, situado na parte externa da interface camada de gel/solvente, representada na Figura 5. A concentração do solvente na matriz aumenta progressivamente e verifica-se diluição das cadeias poliméricas em virtude do aumento das interações águapolímero. Nesta camada limite, ainda existem alguns pontos de interação entre as cadeias poliméricas e as partículas de fármaco circulem entre elas.

Finalmente, o polímero assume a terceira configuração macromolecular, designada por tipo diluído (Figura 7C). Nesta configuração, as cadeias poliméricas estão completamente separadas umas das outras e dispersas no líquido de dissolução, perfeitamente individualizadas e movendose livremente com movimentos Brownianos. Neste momento, completa-se a liberação do fármaco restante ainda contido no interior da matriz para o líquido de dissolução, que ocorre na etapa 6 da Figura 4.

\section{CINÉTICAS PARA ANÁLISE DA LIBERAÇÃO IN VITRO}

A etapa de liberação da substância ativa a partir de

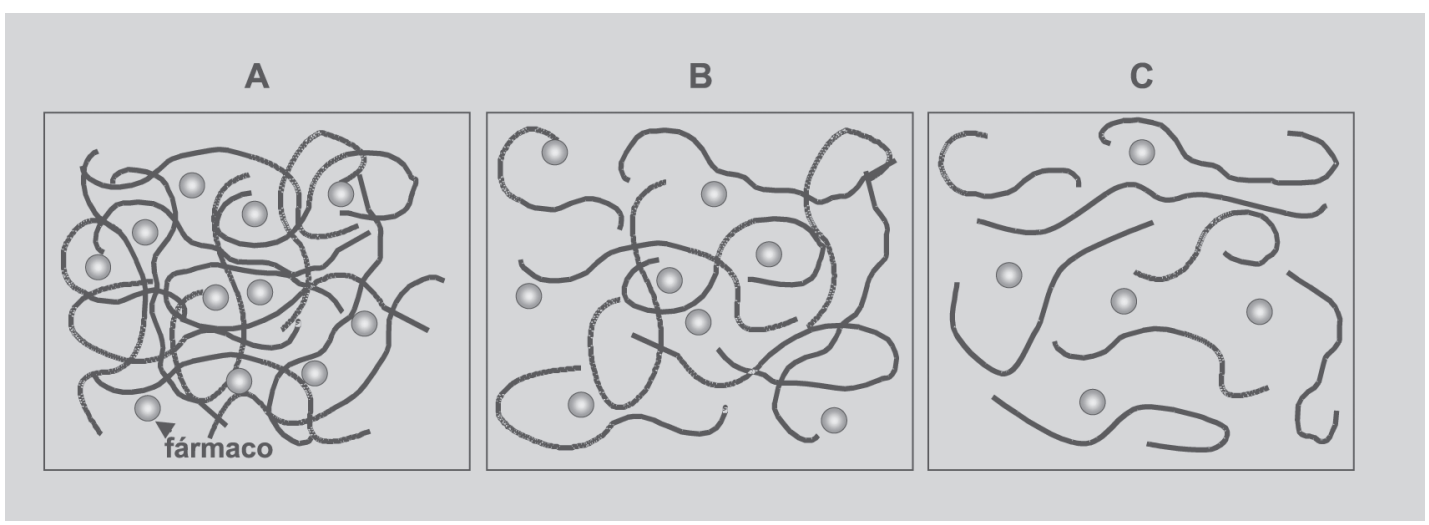

FIGURA 7 - Configurações macromoleculares dos polímeros hidrofílicos durante o processo de dissolução do fármaco nos sistemas matriciais (adaptado de Narasimhan e Peppas, 1997). 
sistemas de matrizes hidrofílicas resulta da interação complexa entre a dissolução, a difusão e os mecanismos de erosão. Esta complexidade deve-se, em parte, ao fato de as moléculas de HPMC, quando em contato com a água e em conseqüência do processo de transição do estado seco para o estado maleável, adquirirem micro e macro-estrutura, cujas configurações, já referidas anteriormente, dependem do tempo de exposição à água. Conseqüentemente, o tratamento matemático, abrangendo todos estes mecanismos, torna-se muito complexo. Um número significativo de trabalhos experimentais e teóricos foram publicados na literatura com o objetivo de elucidar o modelo de transporte do fármaco e revelar quais os mecanismos de liberação do fármaco destes sistemas matriciais (Lee, Kim, 1991; Hariharan et al., 1994; Colombo et al., 1995,1996, 2000; Ferrero et al., 2000; Kiil, Dam-Johansen, 2003). Recentemente, Siepmann e Peppas (2001) publicaram uma revisão detalhada dos vários tipos de modelos matemáticos desenvolvidos para os sistemas constituídos à base do polímero HPMC. Esta compilação inclui desde correlações empíricas e semi-empíricas simples e facilmente aplicáveis até formulas mais sofisticadas, as quais se considera o tamanho e a forma geométrica das matrizes poliméricas.

Algumas características físicas do fármaco incorporado e da matriz de HPMC (como a solubilidade em água, as densidades e a porosidade da matriz inicial) aparentemente não aparecem explícitas nos modelos desenvolvidos. Por exemplo, as características de dissolução (solubilidade e velocidade de dissolução) devem ser tomadas em atenção no caso de fármacos fracamente solúveis em água (Siepmann, Peppas, 2000). Se a concentração de fármaco excede a solubilidade, as formas dissolvida e não dissolvida de fármaco co-existem na matriz. É importante considerar que somente o fármaco dissolvido contribui para a difusão. Assim, os modelos podem ser aplicados apenas em situações específicas e que obedeçam a condições bem definidas. Conseqüentemente, é ainda necessário realizar mais trabalhos de investigação antes de os mecanismos de liberação, a partir de sistemas matriciais de HPMC, estarem completamente elucidados.

Apesar da complexidade dos fenômenos envolvidos na liberação de fármacos a partir de sistemas matriciais hidrofílicos, alguns modelos bem conhecidos são usados extensivamente para analisar a liberação da substância ativa a partir destes sistemas (Costa, Sousa Lobo, 2001).

O primeiro modelo, designado por cinética de ordem zero baseia-se na liberação lenta da substância ativa a partir de formas farmacêuticas que não se desagregam. Este modelo pode ser expresso pela seguinte expressão:

$$
\frac{M_{t}}{M_{\infty}}=K_{0} t+b \quad \text { Equação } 1
$$

na qual $M_{t}$ representa a quantidade absoluta de fármaco liberada no tempo $t$ e $M_{\infty}$ a quantidade total de fármaco liberado num tempo infinito, a qual deverá corresponder à quantidade total de fármaco incorporado ao sistema polimérico no $t=0 ; K_{0}$ é uma constante cinética e $b$ é a quantidade inicial de fármaco na solução. Muitas vezes, esta quantidade inicial do fármaco resulta de uma liberação imediata ("burst effect") motivada ou pela liberação do fármaco existente à superfície do sistema matricial ou por alterações que se verificam na estrutura do sistema com conseqüente liberação imediata do fármaco seguido de liberação mais lenta. Este modelo é geralmente utilizado para descrever a liberação por vários tipos de formas farmacêuticas de liberação controlada, como é o caso dos comprimidos matriciais, dos sistemas osmóticos e das formas revestidas (Varelas et al., 1995). De maneira ideal, as preparações destinadas a veicular substâncias ativas segundo liberação prolongada apresentam um perfil de liberação de ordem zero, verificando-se que a velocidade de difusão do fármaco, do interior para o exterior da matriz, é menor que a respectiva velocidade de dissolução, formando uma solução saturada, que permite a cedência constante do fármaco. Esta situação de liberação ideal é muito difícil de obter na prática. A aplicação deste modelo apresenta muitas limitações devido aos poucos fatores de ajuste ao modelo.

Outro modelo proposto baseia-se na equação de Higuchi (1961), freqüentemente utilizada para descrever a velocidade de liberação controlada do fármaco a partir de um sistema matricial. A Equação 2 representa a equação de Higuchi expressa como fração de massa liberada:

$$
\frac{M_{t}}{M_{\infty}}=K_{H} \sqrt{t}+b
$$

onde $K_{H}$ corresponde à constante de liberação de Higuchi, que reflete as características do desenho da formulação.

Higuchi descreve o mecanismo de liberação dos fármacos como um processo de difusão baseado na lei de Fick, estando dependente da raiz quadrada do tempo. Porém, o uso desta relação em sistemas que intumescem pode tornar-se insuficiente, pois sistemas deste tipo podem ser erodíveis, devendo-se atender ao atributo do relaxamento das cadeias poliméricas para o transporte do fármaco. Assim, a equação de Higuchi apresenta fortes limitações na interpretação dos mecanismos de liberação controlada. No entanto, é mais realista dos vários mecanismos que ocorrem 
nos sistemas matriciais que o modelo de ordem zero. Este modelo pode-se aplicar com maior exatidão a matrizes unidimensionais pouco solúveis, que não apresentem capacidade de intumescimento, como por exemplo o acetato de celulose, nos quais está incorporado um fármaco muito solúvel (Tahara et al., 1996). Para outros sistemas, tais como matrizes constituídas por HPMC, também se utiliza esta equação. No entanto, quando se pretende ter idéia mais exata dos mecanismos de liberação é necessário atender outros fatores físico-químicos.

Outro modelo baseia-se na equação semi-empírica proposta por Korsmeyer et al. (Korsmeyer, Peppas, 1981; Korsmeyer et al., 1983). Esta equação é utilizada para descrever a liberação do soluto quando o mecanismo que prevalece é uma combinação da difusão do fármaco (transporte Fickiano) e do transporte Caso II (nãoFickiano, controlado pelo relaxamento das cadeias poliméricas) (Ritger, Peppas, 1987). Neste modelo, a relação entre a velocidade de liberação e o tempo é igual a:

$$
\frac{M_{t}}{M_{\infty}}=K t^{n}+b \quad \text { Equação } 3
$$

em que $K$ é uma constante cinética, que incorpora características estruturais e geométricas do mecanismo e $n$ é o expoente de liberação que, de acordo com o valor numérico que assume, caracteriza o mecanismo de liberação do fármaco.

Esta equação é geralmente utilizada para interpretar e descrever a liberação do fármaco quando o mecanismo que prevalece não é bem conhecido ou resulta da combinação de dois processos aparentemente independentes: um devido ao transporte do fármaco que obedece às leis de Fick ou transporte Fickiano, e outro conseqüência dos fenômenos de inchamento/relaxamento do gel (expansão dinâmica) e que envolve a transição de um estado semirígido a outro mais flexível, chamado transporte Caso-II. Nesta equação tem-se em conta não só a difusão do fármaco, mas também o relaxamento do gel como processos determinantes na liberação do fármaco.

Consoante a forma geométrica da preparação, assim variam os valores de $n$ usados para interpretar e caracterizar o mecanismo de liberação. O cilindro é a forma geométrica mais comum dos comprimidos; neste caso, quando $n$ é igual a 0,45 , o mecanismo principal, que controla a liberação do fármaco no sistema é a difusão pura (difusão Fickiana clássica). Quando $n$ assume o valor 0,89 , a equação corresponde a cinética de liberação de ordem zero, sendo a liberação controlada por mecanismo de transporte de Caso II, ou seja, pelo fenômeno de intumescimento do polímero (relaxamento da matriz ou liberação mediante erosão). Outros valores de $\mathrm{n}(0,45<\mathrm{n}<0,89)$ indicam cinética de transporte anômalo, ou seja, uma combinação dos dois mecanismos de difusão e de transporte de Caso II.

Nos sistemas de liberação controlada, a extensão e a velocidade de liberação do fármaco resulta da combinação da difusão com o transporte de Caso II das moléculas de fármaco através das cadeias poliméricas (Ritger, Peppas, 1987). Nestes casos, a difusão obedece às leis de Fick, enquanto o transporte de Caso II (Enscore et al., 1977) reflete a influência do relaxamento do polímero no movimento das moléculas na matriz.

Um outro modelo matemático de referência, no caso de sistemas matriciais de HPMC, é o modelo de Peppas e Sahlin (1989). Numa tentativa de quantificar as contribuições relativas dos dois fenômenos responsáveis pela liberação (difusão e relaxamento), Peppas et al. introduziram um segundo termo na equação de KorsmeyerPeppas, surgindo o modelo de Peppas e Sahlin:

$$
\frac{M_{t}}{M_{\infty}}=K_{1} t^{m}+K_{2} t^{2 m} \quad \text { Equação } 4
$$

em que $K_{I}$ e $K_{2}$ representam constantes que refletem as contribuições relativas do mecanismo de difusão Fickiana e do mecanismo de erosão/relaxamento (transporte Caso II) e o coeficiente $m$ é o expoente de difusão Fickiana da preparação farmacêutica que apresente uma liberação modificada, qualquer que seja a sua forma.

Os modelos de ordem zero e o modelo de Higuchi são mutuamente exclusivos: quando se aplica um, o outro já não tem aplicação. O mesmo não sucede com o modelo de Korsmeyer - Peppas e o modelo de Peppas e Sahlin, sendo a aplicação destes modelos bastantes úteis para complementar as informações relativamente aos mecanismos de liberação dos fármacos obtidas com a aplicação dos outros dois modelos.

\section{CONCLUSÃO}

São bem conhecidas as vantagens que as formas farmacêuticas de liberação modificada apresentam relativamente às formas de liberação imediata do mesmo fármaco. Existem várias estratégias para delinear uma forma farmacêutica de liberação modificada. A estratégia mais comum, principalmente por apresentar uma tecnologia de fabricação pouco complicada e de baixo custo, é a preparação de comprimidos matriciais. Entre estes sistemas, os compostos que constituem matrizes hidrofílicas, como os éteres da celulose são amplamente utilizados, principalmente a HPMC. As características dos polímeros hidrofílicos e a sua capacidade de responder à 
presença de água e formar uma camada de gel são essenciais para controlar a liberação destas matrizes.

A escolha do tipo de polímero hidrofílico e da quantidade presente na formulação da matriz é essencial para obter combinação adequada dos vários mecanismos (intumescimento, dissolução ou erosão) provavelmente envolvidos na cinética da liberação in vitro. A importância da melhor compreensão destes mecanismos reside, entre outras vantagens, na possibilidade de modulação e de um controle mais eficaz da liberação de fármacos incorporados no sistema matricial.

\section{ABSTRACT \\ Modified release of drug delivery systems: hydrophilic polymers}

Drug delivery systems (DDS) became an integral part of pharmaceutical research. The majority of oral DDS are matrix-based systems. Hydrophilic matrices for the past two decades have been popular in the formulation of controlled release solid dosage forms. Swellable matrices are monolithic systems prepared by compression of a powdered mixture of a hydrophilic polymer and a drug. They represent a delivery system in which various mechanisms can be adapted to the delivery program. Their success is linked to the established tabletting technology of manufacture. The choice of the hydrophilic polymer in the matrix formulation can provide an appropriate combination of swelling, dissolution or erosion mechanisms to evaluate in vitro release kinetics.

UNITERMS: Controlled release. Matrix polymers. Hydrophilic matrices.

\section{AGRADECIMENTOS}

Carla Lopes agradece o apoio financeiro à FCT (Fundação para a Ciência e Tecnologia) pela bolsa de doutoramento (SFRH/BD/7002/2001).

\section{REFERÊNCIAS BIBLIOGRÁFICAS}

BETTINI, R., COLOMBO, P., MASSIMO, G., SANTI, P., CATELLANI, P.L., PEPPAS, N.A. Moving fronts and drug release from hydrophilic matrices. INTERNATIONAL SYMPOSIUM ON CONTROLLED RELEASE OF BIOACTIVE MATERIALS. 21., Nice, France, 1994. Proceedings. Deerfield: Controlled Release Society, 1994. v. 21, p. 19-20.
BETTINI, R., COLOMBO, P., PEPPAS, N.A. Drug concentration profiles during release from hydrophilic matrices. INTERNATIONAL SYMPOSIUM ON CONTROLLED RELEASE OF BIOACTIVE MATERIALS. 24., Stockholm, Sweden, 1997. Proceedings. Deerfield: Controlled Release Society, 1997. v. 24, p. 635-636.

BETTINI, R., PEPPAS, N.A., COLOMBO, P. Polymer relaxation in swellable matrices contributes to drug release”. INTERNATIONAL SYMPOSIUM ON CONTROLLED RELEASE OF BIOACTIVE MATERIALS. 25., Las Vegas, Nevada, USA, 1998. Proceedings. Deerfield: Controlled Release Society, 1998. v. 25, p. 36-37.

BURI, P. Définition et classification des systèmes matriciels. S. T. P. Pharma., v. 3, p. 193-199, 1987.

BURI, P.; DOELKER, E. Formulation des comprimés à libération prolongée II. Matrices hydrophiles. Pharm. Acta Helv., Amsterdam, v. 55, n. 7-8, p. 189-197, 1980.

COLOMBO, P.; BETTINI, R.; MASSIMO, G.; CATELLANI, P.L.; SANTI, P.; PEPPAS, N.A. Drug diffusion front movement is important in drug release control from swellable matrix tablets. J. Pharm. Sci., New York, v. 84, n. 8, p. 991-997, 1995.

COLOMBO, P.; BETTINI, R.; PEPPAS, N.A. Observation of swelling process and diffusion front position during swelling in hydroxylpropyl methylcellulose matrices containing a soluble drug. J. Control. Release, Amsterdam, v. 61, n. 1-2, p. 83-91, 1999.

COLOMBO, P.; BETTINI, R.; SANTI, P. Swellable matrices for controlled drug delivery: gel-layer behaviour, mechanisms and optimal performance. PSTT, New York, v. 3, n. 6, p. 198-204, 2000.

COLOMBO, P.; BETTINI, R.; SANTI, P.; DE ASCENTIS, A.; PEPPAS, N.A. Analysis of the swelling and release mechanisms from drug delivery systems with emphasis on drug solubility and water transport. J. Control. Release, Amsterdam, v. 39, n. 2-3, p. 231-237, 1996.

COSTA, P.; SOUSA LOBO, J.M. Formas farmacêuticas de libertação modificada. Rev. Port. Farm., Lisboa, v. 59, n. 4, p. 181-190, 1999.

COSTA, P.; SOUSA LOBO, J.M. Modelling and comparison of dissolution profiles. Eur. J. Pharm. Sci., Amsterdam, v. 13, n. 2, p. 123-133, 2001. 
DAS, N. G.,; DAS, S.K. Controlled-release of oral dosage forms. Formulation, Fill \& Finish - a Technology Primer 2003 - a supplement to Pharm. Tec., v. 27, p. 10-16, 2003. Disponível em: $<$ http://www.pharmtech.com/pharmtech/ data/articlestandard/pharmtech/232003/59302/ article.pdf $>$ Acesso em: 17 ago. 2004.

DOELKER, E. Cellulose derivatives. Adv. Polym. Sci., Berlin, v. 107, p. 199-265, 1993.

ENSCORE, D.; HOPFENBERG, H.; STANNETT, V. Effect of particle size on the mechanism controlling nhexane in glassy polystyrene microspheres. Polymer, Amsterdam, v. 18, n. 8, p. 793-800, 1977.

EYJOLFSSON, R. Hydroxypropyl methylcellulose mixtures: effects and kinetics of release of an insoluble drug. Drug Dev. Ind. Pharm., New York, v. 25, n. 5, p. 667-669, 1999.

FEELY, L. S.; DAVIS, S. S. The influence of surfactants on drug release from hydroxypropylmethylcellulose matrices. Int. J. Pharm., Amsterdam, v. 41, n. 1-2, p. 8390, 1988a.

FEELY, L. S.; DAVIS, S. S. The influence of polymeric excipients on drug release from hydroxypropylmethylcellulose matrices. Int. J. Pharm., Amsterdam, v. 44, n. $1-3$, p. 131-139, 1988 b.

FERRERO, C.; MUÑOZ-RUIZ, A.; JIMÉNEZCASTELLANOS, M.R. Fronts movement as a useful tool for hydrophilic matrix release mechanism elucidation. Int. J. Pharm., Amsterdam, v. 202, n. 1-2, p. 21-28, 2000.

FORD, J.L.; RUBINSTEIN, M.H.; HOGAN, J.E. Formulation of sustained release promethazine hydrochloride tablets using hydroxypropylmethylcellulose matrices. Int. J. Pharm., Amsterdam, v. 24, n. 2-3, p. 337$338,1985$.

FORD, J.L.; RUBINSTEIN, M.H.; McCAUL, F.; HOGAN, J.E.; EDGAR, P.J. Importance of drug type, tablet shape and added diluents on drug release kinetics from hydroxypropylmethylcellulose matrix tablets. Int. J. Pharm., Amsterdam, v. 40, n. 3, p. 223-234, 1987.

FYFE, C. A.; BLAZED, A. I. Investigation of hydrogel formation from hydroxypropylmethylcellulose (HPMC) by NMR spectroscopy and NMR imaging techniques. Macromolecules, Easton, v. 30, n. 20, p. 6230-6237, 1997.
GAO, P.; FAGERNESS, P.E. Diffusion in HPMC gels. I. Determination of drug and water diffusity by pulsed-fieldgradient spin-echo NMR. Pharm. Res., New York, v. 12, n. 7, p. 955-964, 1995.

GAO, P.; SKOUG, J.W.; NIXON, P.R.; JU, T.R.; STEMM, N.L.; SUNG, K.C. Swelling of hydroxypropyl methylcellulose matrix tablets. 2. Mechanistic study of the influence of formulation variables on matrix performance and drug release. J. Pharm. Sci., New York, v. 85, n. 7 , p. 732-740, 1996.

HARIHARAM, D.;PEPPAS, N.A.; BETTINI, R.; COLOMBO, P. Mathematical analysis of drug delivery from swellable systems with parcial physical restrictions or impermeable coatings. Int. J. Pharm., Amsterdam, v. 112, n. 1, p. 47-54, 1994.

HIGUCHI, T. Rate of release of medicaments from ointment bases containing drugs in suspension. J. Pharm. Sci., New York, v. 50, p. 874-875, 1961.

JUANG, R.H.; STOREY, D. Correlation of characteristics of gel extrusion module (GEM) tablet formulation and drug dissolution rate. J. Control. Release, Amsterdam, v. 89, n. 3, p. 375-385, 2003.

KIIL, S.; DAM-JOHANSEN, K. Controlled drug delivery from swellable hydroxypropylmethylcellulose matrices: Model-based analysis of observed radial front movements. J. Control. Release, Amsterdam, v. 90, n. 1, p. 1-21, 2003.

KORSMEYER, R.W.; GURNY, R.; DOELKER, E.; BURI, P.; PEPPAS, N.A. Mechanisms of solute release from porous hydrophilic polymers. Int. J. Pharm., Amsterdam, v. 15, n. 1, p. $25-35,1983$.

KORSMEYER, R.W.; PEPPAS, N.A. Macromolecular and modeling aspects of swelling-controlled systems. In: ROSEMAN, T.J.; MANSDORF, S.Z., (Eds.). Controlled release delivery systems. New York: Marcel Dekker Inc., 1981. p. 77-90.

LEE, P.I.; KIM, C. Probing the mechanisms of drug release from hydrogels. J. Control. Release, Amsterdam, v. 16, n. 1-2, p. 229-236, 1991.

LORDI, N.G. Formas farmacêuticas de liberação prolongada In: LACHMAN, L.; LIEBERMAN, H. A.; KANIE, J. L. (Eds.). Teoria e prática na indústria farmacêutica. Lisboa: Ed. Fundação Calouste Gulbenkia, 2001. v. II, p. 737-781. 
NARASIMHAN, B.; PEPPAS, N.A. Molecular analysis of drug delivery systems controlled by dissolution of the polymer carrier. J. Pharm. Sci., New York, v. 86, n. 3, p. 297-304, 1997.

PHAM, A.T.; LEE, P.I. Probing the mechanism of drug release from hydroxypropyl methylcellulose matrices. Pharm. Res., New York, v. 11, n. 10, p. 1379-1384, 1994.

PEPPAS, N.A.; BURES, P.; LEOBANDUNG, W.; ICHIKAWA, H. Hydrogeles in pharmaceutical formulations. Eur. J. Pharm. Biopharm., Amsterdam, v. 50, n. 1, p. 27-46, 2000.

PEPPAS, N.A.; SAHLIN, J.J. A simple equation for the description of solute release. III. Coupling of diffusion and relaxation. Int J. Pharm., Amsterdam, v. 57, n. 2, p.169$172,1989$.

RANGA RAO, K.V.; DEVI, K.P. Swelling controlled release systems: recent development and application. Int. J. Pharm., Amsterdam, v. 48, n. 1-3, p.1-13, 1988.

RITGER, P.; PEPPAS, N.A. A simple equation for description of solute release. II. Fickian and anomalous release from swellable devices. J. Control. Release, Amsterdam, v. 5, n. 1, p. 37-42, 1987.

RODRIGUEZ, C.F., BRUNEAU, N., BARRA, J., ALFONSO, D., DOELKER, E. Hydrophilic cellulose derivatives as drug delivery carriers: influence of substitution type on the properties of compressed matrix tablets. In: WISE, D.L., (Ed.). Handbook of Pharmaceutical Controlled Release Technology. New York: Marcel Dekker, Inc, 2000.

SALOMON, J.L.; DOELKER, E. Formulation of sustained release tablets. I. Inert matrices. Pharm. Acta Helv., Amsterdam, v. 55, p.174-182, 1980.

SHESKEY, P.J.; CABELKA, T.D. Reworkability of sustained-release tablet formulations containing HPMC polymers. Pharm. Technol., New York, v. 7, p. 60-74, 1992.

SHESKEY, P.J.; CABELKA, T.D.; ROBB, R.T., BOYCE, B.M. Use of roller compaction in the preparation of controlled-release hydrophilic matrix tablets containing methylcellulose and hydroxypropyl methylcellulose polymers. Pharm. Technol., New York, v. 9, p. 133-150, 1994
SIEPMANN, J.; PEPPAS, N.A. Modelling of drug release from delivery systems based on hydroxypropyl methylcellulose (HPMC). Adv. Drug Deliv. Rev., New York, v. 48, n. 2-3, p. 139-157, 2001.

SIEPMANN, J.; PEPPAS, N.A. Hydrophilic matrices for controlled drug delivery: an improved mathematical model to predict the resulting drug delivery kinetics (the "sequential layer" model). Pharm. Res., New York, v. 17, n. 10, p. 1290-1298, 2000.

SKOUG, J.W.; MIKELSONS, M.V.; VIGNERON, C.N.; STEMM, N.L. Qualitative evaluation of the mechanism of release of matrix sustained release dosage forms by measurement of polymer release. J. Controlled Release, Amsterdam, v. 27, n. 3, p. 227-245, 1993.

TAHARA, K.; YAMAMOTO, K.; NISHIHATA, T. Application of model-independent and model analysis for the investigation of effect of drug solubility on its release rate from hydroxypropyl methylcellulose sustained release tablets. Int. J. Pharm., Amsterdam, v. 133, n. 1, p. 17-27, 1996.

TAHARA, K.; YAMAMOTO, K.; NISHIHATA, T. Overall mechanism behind matrix sustained release (SR) tablets prepared with hydroxypropyl methylcellulose. $J$. Controlled Release, Amsterdam, v. 35, n. 1, p. 59-66, 1995.

VARELAS, C.G.; DIXON, D.G.; CAROL, S. Zero-order release from biphasic polymer hydrogels. J. Controlled Release, Amsterdam, v. 34, n. 3, p. 185-192, 1995.

WAN, L.S.C.; HENG, P.W.S.;WONG, L.F. The effect of hydroxypropyl methylcellulose on water penetration into a matrix system. Int. J. Pharm., Amsterdam, v. 73, n. 2, p. 111-116, 1991.

WAN, L.S.C.; HENG, P.W.S.; WONG, L.F. Matrix swelling: a simple model describing extent of swelling of HPMC matrices. Int. J. Pharm., Amsterdam, v. 116, n. 2, p. 159168, 1995.

WESSENLINGH, J.A. Controlling diffusion. J. Controlled Release, Amsterdam, v. 24, n. 1-3, p. 47-60, 1993.

Recebido para publicação em 21 de setembro de 2004 Aceito para publicação em 04 de março de 2005 